\title{
A NUMERICAL STUDY OF THE OPTIMAL CONTROL PROBLEM FOR DEGENERATE MULTICOMPONENT MATHEMATICAL MODEL OF THE PROPAGATION OF A NERVE IMPULSE IN THE SYSTEM OF NERVES
}

\author{
O. V. Gavrilova, South Ural State University, Chelyabisk, Russian Federation, \\ gavrilovaov@susu.ru
}

\begin{abstract}
The article is devoted to a numerical study of optimal regulation of propagation of a nerve impulse in the system of nerves, which can be constructed on the basis of the optimal control problem for degenerate FitzHugh - Nagumo system of equations. This model belongs to the class of reaction-diffusion models, which model a wide class of processes such as chemical reactions with diffusion and the propagation of a nerve impulse. In the case of asymptotic stability of the considered problem and under assumption that the rate of change of one component significantly exceeds the rate of the others, the model under study can be reduced to the optimal control problem for a semilinear Sobolev type equation with the initial Showalter - Sidorov condition. The article develops an algorithm for the numerical study of the model in the Maple environment. This algorithm is based on the Galerkin method and the decomposition method, which allows to take into account the phenomenon of degeneracy of the equation. The article gives an example illustrating the results of the computational experiment obtained by the two-component model on the two-ribbed graphs.

Keywords: Sobolev type equations; Showalter - Sidorov problem; optimal control problem.
\end{abstract}

\section{Introduction}

Mathematical model of the propagation of a nerve impulse in a membrane belongs to the class of reaction-diffusion models, which is a large class of processes [1-4]. For instance, the study of the Belousov - Zhabotinsky chemical reaction, creating ordered temporal and/or spatial structures is based on the reaction-diffusion equations. Reaction-diffusion models describe various chemical and biological processes. In the works [1-4], researchers study only two-component reaction-diffusion models. At the present time, the studies of more complex temporal and spatial structures lead to research of multicomponent reactiondiffusion model $[5,6]$. Most often, models with only one activator and several inhibitors are considered [5]. In these models, the multicomponent is usually achieved by increasing the number of inhibitors. In [5], three- and four-component models (one activator and two (three) inhibitors were considered) and their stability were studied. The necessity to study the degenerate case is related to the fact that the rate of change of ones of concentration is much greater than that of the others. The degenerate system of reaction-diffusion equations was first studied in the article [7].

A multicomponent model of the propagation of a nerve impulse in the system of nerves can be defined as a FitzHugh - Nagumo system on the finite connected oriented graph 
$\mathbf{G}=\mathbf{G}(\mathcal{V} ; \mathcal{E})$ with the vertex set $\mathcal{V}=\left\{V_{h}\right\}_{h=1}^{M}$ and the edge set $\mathcal{E}=\left\{E_{j}\right\}_{j=1}^{K}$, i.e.

$$
\left\{\begin{array}{c}
v_{1 j t}-\alpha_{1} v_{1 j s s}+\beta_{11} v_{1 j}+\beta_{12} v_{2 j}+\cdots+\beta_{1 m} v_{m j}+\left(v_{1 j}\right)^{3}=u_{1 j} \\
v_{2 j t}-\alpha_{2} v_{2 j s s}+\beta_{21} v_{1 j}+\beta_{22} v_{2 j}+\cdots+\beta_{2 m} v_{m j}+\left(v_{2 j}\right)^{3}=u_{2 j} \\
\cdots \\
v_{k j t}-\alpha_{k} v_{k j s s}+\beta_{k 1} v_{1 j}+\beta_{k 2} v_{2 j}+\cdots+\beta_{k m} v_{m j}+\left(v_{k j}\right)^{3}=u_{k j} \\
-\alpha_{k+1} v_{(k+1) j s s}+\beta_{(k+1) 1} v_{1 j}+\beta_{(k+1) 2} v_{2 j}+\cdots+\beta_{(k+1) m} v_{m j}=u_{(k+1) j} \\
\cdots \\
-\alpha_{m} v_{m j s s}+\beta_{m 1} v_{1 j}+\beta_{m 2} v_{2 j}+\cdots+\beta_{m m} v_{m j}=u_{m j} \\
\text { for all } s \in\left(0, l_{j}\right), j=\overline{1, K}
\end{array}\right.
$$

where the positive parameters $\alpha_{i}, i=\overline{1, m}$, are the diffusion coefficients and the prescribed functions $u_{i j}=u_{i j}(s, t), i=\overline{1, m}, j=\overline{1, K}$, characterize exterior actions. Each function $v_{i j}=v_{i j}(s, t)$ satisfies the flow balance condition

$$
\sum_{j: E_{j} \in E^{\alpha}\left(V_{h}\right)} d_{i j} v_{i j s}(0, t)-\sum_{r: E_{r} \in E^{\omega}\left(V_{h}\right)} d_{i r} v_{i r s}\left(l_{r}, t\right)=0,
$$

and the continuity condition

$$
v_{i r}(0, t)=v_{i j}(0, t)=v_{i q}\left(l_{q}, t\right)=v_{i n}\left(l_{n}, t\right)
$$

at each vertex $V_{h}$ for $h=\overline{1, M}$ for all $E_{r}, E_{j} \in E^{\alpha}\left(V_{h}\right)$ and $E_{q}, E_{n} \in E^{\omega}\left(V_{h}\right)$. Here $E^{\alpha(\omega)}\left(V_{h}\right)$ stands for the set of edges starting (ending) at $V_{h}$.

In the special constructed Banach spaces $\mathfrak{X}$ and $\mathfrak{U}$, the preimage of degenerate system (1) is the abstract semilinear Sobolev-type equation

$$
\frac{d}{d t} L x+M(x)=u, \text { ker } L \neq\{0\}
$$

Here $L$ is a continuous linear operator and $M$ is a smooth nonlinear operator. Endow equation (4) with the Showalter-Sidorov condition

$$
L\left(x(0)-x_{0}\right)=0 .
$$

Sobolev-type equations on a graph were studied for the first time in [8], where sufficient conditions for the simplicity of a phase manifold of the Hoff equation were found as well.

The mathematical model of optimal regulation of the propagation of a nerve impulse in the system of nerves can be constructed on the basis of the optimal control problem

$$
J(x, u) \rightarrow \inf
$$

by the solutions to $(1)-(3)$ in the weak generalized sense. Here $J(x, u)$ is a certain purpose-built quality functional with the control $u \in \mathfrak{U}_{a d}$, where $\mathfrak{U}_{a d}$ is a closed convex set in the control space $\mathfrak{U}$. The optimal control problem for linear Sobolev-type equations with the Cauchy initial condition was originally posed and studied in [9]. That article initiated a series of studies of optimal control problems for linear Sobolev-type equations with various initial conditions [10,11]. Sufficient conditions for the existence of a solution to problem $(4),(5),(6)$ when $L$ is a Fredholm operator were obtained in [12,13]. The optimal 
control problems in various reaction-diffusion models are studied for non-degenerate cases in the work [14] and for deganerate cases in the works $[15,16]$. The optimal control problem for degenerate two-component FitzHugh-Nagumo model on the domain $\Omega$ and the Showalter-Sidorov problem are considered in [15]. The article [16] proves the existence and uniqueness of a solution to the Showalter-Sidorov problem in the weak generalized sense and the existence of optimal control by weak generalized solutions to this problem and finds sufficient conditions for the existence of optimal control problem for a multicomponent mathematical model of the propagation of a nerve impulse in system of nerves (1) - (3), (5), (6).

Our goal is to construct an algorithm for the numerical study of optimal regulation of the propagation of a nerve impulse in system of nerves $(1)-(3),(5),(6)$. The need to develop a numerical solution algorithm is due to the fact that it is not always possible to obtain an analytical solution for degenerate reaction-diffusion models. Therefore, the construction of algorithms for numerical methods of the studied problems is in demand. In this case, the Galerkin method is the most suitable as the method that allows to take into account the degeneracy of the equations for certain parameters. Using the Galerkin method, we can construct approximate solutions of models, which coefficients satisfy a system of algebra-differential equations with the corresponding initial conditions [17]. This article develops an algorithm based on the modified Galerkin method for degenerate equations on a finite connected oriented graph. Based on the ideas and methods described in $[12,18]$, we present the desired functions in the form of the Galerkin sum $\tilde{v}_{i}(s, t)=$ $\sum_{l=1}^{n} v_{i}^{l}(t) \varphi_{l}(s)$, where $\left\{\varphi_{l}(s)\right\}$ are the eigenfunctions of the homogeneous Dirichlet problem of the Laplace operator $\Delta$ in the graph $\mathbf{G}$, and $v_{i}^{l}(t)$ satisfy the system of algebraic-differential equations and the corresponding initial conditions. Here it is necessary to mention the decomposition method, which is described in [12], and, along with the Galerkin method, is the basis of computational experiments. Using all of the above methods, we study system of equations (1) - (3) on the finite connected oriented graph G with Showalter-Sidorov condition (5). In order to illustrate the results obtained, we give examples of the twocomponent model on the two-ribbed graphs.

\section{Statement of the Problem}

Consider the finite connected oriented graph $\mathbf{G}=\mathbf{G}(\mathcal{V} ; \mathcal{E})$ with the vertex set $\mathcal{V}=$ $\left\{V_{h}\right\}_{h=1}^{M}$ and the edge set $\mathcal{E}=\left\{E_{j}\right\}_{j=1}^{K}$, where each edge has the length $l_{j}>0$ and the transverse cross-section area $d_{j}>0$. On $\mathbf{G}$, consider FitzHugh-Nagumo system (1), where each function $v_{i j}=v_{i j}(s, t)$ satisfies flow balance condition (2) and continuity condition (3) at each vertex $V_{h}$ for $h=\overline{1, M}$, for all $E_{r}, E_{j} \in E^{\alpha}\left(V_{h}\right)$ and $E_{q}, E_{n} \in E^{\omega}\left(V_{h}\right)$. Conditions (2), (3) and system (1) form the multicomponent mathematical model of neural signal propogation. Endow (2), (3) with the Showalter-Sidorov conditions

$$
v_{i}(s, 0)=v_{0 i}(s), i=\overline{1, k} .
$$

Based on the results obtained in [8], introduce the Banach space

$$
L_{p}(\mathbf{G})=\left\{g=\left(g_{1}, g_{2}, \ldots, g_{j}, \ldots, g_{K}\right): g_{j} \in L_{p}\left(0, l_{j}\right)\right\} .
$$

Construct the Banach space

$$
\mathfrak{H}=\left\{g=\left(g_{1}, g_{2}, \ldots, g_{j}, \ldots, g_{K}\right): g_{j} \in W_{2}^{1}\left(0, l_{j}\right) \text { and condition (3) holds }\right\}
$$


Consider the Hilbert space $\mathcal{H}=\left(L_{2}(\mathbf{G})\right)^{m}$ endowed with the inner product $[x, \zeta]=$ $\sum_{i=1}^{m}\left\langle v_{i}, \zeta_{i}\right\rangle, x=\left(v_{1}, v_{2}, \ldots, v_{m}\right), \zeta=\left(\zeta_{1}, \zeta_{2}, \ldots, \zeta_{m}\right), v_{i} \in L_{2}(\mathbf{G}), \zeta_{i} \in L_{2}(\mathbf{G})$, where $\langle\cdot, \cdot\rangle$ is an inner product in the Hilbert space $L_{2}(\mathbf{G})$ identified with its adjoint one. By analogy, construct the space $\mathfrak{A}=\mathfrak{H}^{m}$ and denote by $\mathfrak{A}^{*}$ the adjoint space to $\mathfrak{A}$ with respect to the inner product in $\mathcal{H}$. Write $u=\left(u_{1}, u_{2}, \ldots, u_{m}\right)$ and define the operators $L$ and $M=M_{1}+M_{2}$ :

$$
\begin{gathered}
{[L x, \zeta]=\left\langle v_{1}, \zeta_{1}\right\rangle+\cdots+\left\langle v_{k}, \zeta_{k}\right\rangle, \quad x, \zeta \in \mathfrak{A}} \\
{\left[M_{1}(x), \zeta\right]=\alpha_{1}\left\langle v_{1 s}, \zeta_{1 s}\right\rangle+\left\langle\beta_{11} v_{1}+\beta_{12} v_{2}+\cdots+\beta_{1 m} v_{m}, \zeta_{1}\right\rangle+\alpha_{2}\left\langle v_{2 s}, \zeta_{2 s}\right\rangle+} \\
+\left\langle\beta_{21} v_{1}+\beta_{22} v_{2}+\cdots+\beta_{2 m} v_{m}, \zeta_{2}\right\rangle+\cdots+\alpha_{m}\left\langle v_{m s}, \zeta_{m s}\right\rangle+ \\
+\left\langle\beta_{m 1} v_{1}+\beta_{m 2} v_{2}+\cdots+\beta_{m m} v_{m}, \zeta_{m}\right\rangle, \quad x, \zeta \in \mathfrak{A}, \\
{\left[M_{2}(x), \zeta\right]=\left\langle v_{1}^{3}, \zeta_{1}\right\rangle+\left\langle v_{2}^{3}, \zeta_{2}\right\rangle+\ldots+\left\langle v_{k}^{3}, \zeta_{k}\right\rangle, \quad x, \zeta \in \mathfrak{A},}
\end{gathered}
$$

where $v_{i}^{3}=\left(v_{i 1}^{3}, v_{i 2}^{3}, \ldots, v_{i K}^{3}\right), i=\overline{1, k}$.

Suppose that the matrix $B=\left\{\beta_{i j}\right\}_{i, j=1}^{m}$ has the property

$$
\exists C_{B}, C^{B}>0: \quad C_{B}[x, x] \leq[B x, x] \leq C^{B}[x, x] .
$$

Lemma 1. [16] (i) The operator $L \in \mathcal{L}\left(\mathfrak{A} ; \mathfrak{A}^{*}\right)$ is self-adjoint and nonnegative definite.

(ii) Suppose that $\alpha_{i} \in \mathbb{R}_{+}$for $i=\overline{1, m}$ and condition (8) is satisfied. Then the operator $\left.M_{1} \in \mathcal{L}\left(\mathfrak{A} ; \mathfrak{A}^{*}\right)\right)$ is s-monotone and 2 -coercive.

(iii) The operator $M_{2} \in C^{\infty}\left(L_{4}(\mathbf{G}) ; L_{\frac{4}{3}}(\mathbf{G})\right)$ is s-monotone and 4-coercive.

Therefore, problem (1) - (3), (7) is reduced to Showalter-Sidorov problem (4), (5).

Remark 1. By construction of $L$, the sets $\operatorname{ker} L$, coim $L$, coker $L$, and im $L$ are defined as

$$
\begin{gathered}
\operatorname{ker} L=\underbrace{\{0\} \times\{0\} \times \cdots \times\{0\}}_{k} \underbrace{\times \mathfrak{H} \times \mathfrak{H} \times \cdots \times \mathfrak{H}}_{m-k}, \\
\operatorname{coim} L=\underbrace{\mathfrak{H} \times \mathfrak{H} \times \cdots \times \mathfrak{H}}_{k} \underbrace{\times\{0\} \times\{0\} \times \cdots \times\{0\}}_{m-k}, \\
\operatorname{coker} L=\underbrace{\{0\} \times\{0\} \times \cdots \times\{0\}}_{m} \underbrace{\times \mathfrak{H}^{*} \times \mathfrak{H}^{*} \times \cdots \times \mathfrak{H}^{*}}_{m-k}, \\
\operatorname{im} L=\underbrace{\mathfrak{H}^{*} \times \mathfrak{H}^{*} \times \cdots \times \mathfrak{H}^{*}}_{m-k} \underbrace{\times\{0\}}_{k\{0\} \times\{0\} \times \cdots \times\{0\}}
\end{gathered}
$$

Construct the spaces

$$
\begin{array}{r}
\mathfrak{X}=\left\{x=\left(v_{1}, v_{2}, \ldots, v_{k}, v_{k+1}, \ldots, v_{m}\right): v_{i} \in L_{\infty}\left(0, T ; L_{4}(\mathbf{G})\right) \cap L_{4}\left(0, T ; L_{4}(\mathbf{G})\right)\right), \\
\left.\left.\frac{d v_{i}}{d t} \in L_{2}(0, T ; \mathfrak{H}), i=\overline{1, k} ; v_{i} \in L_{\infty}(0, T ; \mathfrak{H}) \cap L_{2}(0, T ; \mathfrak{H})\right), i=\overline{k+1, m}\right\} ; \\
\mathfrak{U}=\left\{u=\left(u_{1}, u_{2}, \ldots, u_{m}\right): u_{i} \in L_{\frac{4}{3}}\left(0, T ; L_{\frac{4}{3}}(\mathbf{G})\right), i=\overline{1, k} ;\right. \\
\left.u_{i} \in L_{2}\left(0, T ; \mathfrak{H}^{*}\right), i=\overline{k+1, m}\right\} .
\end{array}
$$


By the Sobolev embedding theorem, the space $W_{2}^{1}\left(0, l_{j}\right)$ consists of absolutely continuous functions, therefore, $\mathfrak{H}$ is well-defined, dense, and compactly embedded into $L_{2}(\mathbf{G})$. Fix $a>0$ and construct the operator

$$
\langle A g, h\rangle=\sum_{E_{j} \in \mathcal{E}} d_{j} \int_{0}^{l_{j}}\left(g_{j s}(s) h_{j s}(s)+a g_{j}(s) h_{j}(s)\right) d s, g, h \in \mathfrak{H} .
$$

The operator $A \in \mathcal{L}\left(\mathfrak{H} ; \mathfrak{H}^{*}\right)$ is bijective, its spectrum is real, discrete, of finite multiplicity, and accumulates only at $+\infty$, while its eigenfunctions form a basis for the space $\mathfrak{H}$ [19]. Denote by $\left\{\varphi_{l}\right\}$ a sequence of eigenfunctions of the homogeneous Dirichlet problem for the operator $A$ on the graph $\mathbf{G}$.

Construct Galërkin approximations to the solution to problem (1) - (3) as

$$
v_{i}^{n}(s, t)=\sum_{l=1}^{n} a_{i}^{l}(t) \varphi_{l}(s), i=\overline{1, m}
$$

where the coefficients $a_{i}^{l}$ are determined by the system

$$
\left\{\begin{aligned}
&\left\langle v_{1 t}^{n}-\alpha_{1} v_{1 s s}^{n}+\beta_{11} v_{1}^{n}+\beta_{12} v_{2}^{n}+\cdots+\beta_{1 m} v_{m}^{n}+\left(v_{1}^{n}\right)^{3}, \varphi_{l}\right\rangle=\left\langle u_{1}, \varphi_{l}\right\rangle, \\
&\left\langle v_{2 t}^{n}-\alpha_{2} v_{2 s s}^{n}+\beta_{21} v_{1}^{n}+\beta_{22} v_{2}^{n}+\cdots+\beta_{2 m} v_{m}^{n}+\left(v_{2}^{n}\right)^{3}, \varphi_{l}\right\rangle=\left\langle u_{2}, \varphi_{l}\right\rangle, \\
& \cdots \\
&\left\langle v_{k t}^{n}-\alpha_{k} v_{k s s}^{n}+\beta_{k 1} v_{1}^{n}+\beta_{k 2} v_{2}^{n}+\cdots+\beta_{k m} v_{m}^{n}+\left(v_{k}^{n}\right)^{3}, \varphi_{l}\right\rangle=\left\langle u_{k}, \varphi_{l}\right\rangle, \\
&\left\langle-\alpha_{k+1} v_{(k+1) s s}^{n}+\beta_{(k+1) 1} v_{1}^{n}+\beta_{(k+1) 2} v_{2}^{n}+\cdots+\beta_{(k+1) m} v_{m}^{n}, \varphi_{l}\right\rangle=\left\langle u_{(k+1)}, \varphi_{l}\right\rangle, \\
& \cdots \\
&\left\langle-\alpha_{m} v_{m s s}^{n}+\beta_{m 1} v_{1}^{n}+\beta_{m 2} v_{2}^{n}+\cdots+\beta_{m m} v_{m}^{n}, \varphi_{l}\right\rangle=\left\langle u_{m}, \varphi_{l}\right\rangle
\end{aligned}\right.
$$

for $i=\overline{1, m}, l=\overline{1, n}$ and the Showalter-Sidorov conditions

$$
\begin{gathered}
\left\langle v_{1}(0)-v_{01}, \varphi_{l}\right\rangle=0, \\
\cdots \\
\left\langle v_{k}(0)-v_{0 k}, \varphi_{l}\right\rangle=0, l=\overline{1, n}, \\
v_{0 i}^{n} \rightarrow v_{0 i} \text { for } n \rightarrow \infty \text { in } \mathfrak{H}, i=\overline{1, k} .
\end{gathered}
$$

Note that equations (9) form a system of algebra-differential equations. Let $T_{n} \in \mathbb{R}_{+}$, $T_{n}=T_{n}\left(x_{0}\right), \mathfrak{H}_{n}=\operatorname{span}\left\{\varphi_{1}, \varphi_{2}, \ldots, \varphi_{n}\right\}, \mathfrak{A}_{n}=\left(\mathfrak{H}_{n}\right)^{m}$. For all $x_{0} \in \mathfrak{H}$, there exists a unique local solution $x^{n} \in C^{r}\left(0, T_{n} ; \mathfrak{A}_{n}\right), r \geq 1$, to problem (9), (10) [8].

Definition 1. By a weak generalized solution to (4) we mean the vector function $x \in \mathfrak{X}$ satisfying the condition

$$
\int_{0}^{T} \varphi(t)\left[\frac{d}{d t} L x+M(x), w\right] d t=\int_{0}^{T} \varphi(t)[u, w] d t, \forall w \in \mathfrak{A}, \forall \varphi \in L_{2}(0, T) .
$$

A solution to equation (4) is called a solution to the Showalter-Sidorov problem whenever the solution satisfies (5).

Theorem 1. [16] Suppose that $\alpha_{i} \in \mathbb{R}_{+}$for $i=\overline{1, m}$ and conditions (8) are satisfied. For the given $x_{0} \in \mathfrak{A}$ and $u \in \mathfrak{U}$, there exists a unique solution $x=\left(v_{1}, v_{2}, \ldots, v_{m}\right) \in \mathfrak{X}$ to 
problem $(1)-(3),(7)$, and besides $x^{n}=\left(v_{1}^{n}, v_{2}^{n}, \ldots, v_{m}^{n}\right) \rightarrow\left(v_{1}, v_{2}, \ldots, v_{m}\right)$ for $n \rightarrow \infty$ in the space $\mathfrak{X}$.

Choose a nonempty closed convex set $\mathfrak{U}_{a d} \subset \mathfrak{U}$. Consider the optimal control problem

$$
J(x, u) \rightarrow \inf
$$

for solutions to problem (1) - (3), (7), where the objective functional is defined as

$$
\begin{aligned}
J(x, u)=\vartheta & \sum_{i=1}^{k} \int_{0}^{T}\left\|v_{i}-z_{i}^{d}\right\|_{L_{4}(\mathbf{G})}^{4} d t+\vartheta \sum_{i=k+1}^{m} \int_{0}^{T}\left\|v_{i}-z_{i}^{d}\right\|_{\mathfrak{A}}^{2} d t+ \\
& +(1-\vartheta) \sum_{i=1}^{k} \int_{0}^{T}\left\|u_{i}\right\|_{L_{\frac{4}{3}}}^{\frac{4}{3}}(\mathbf{G}) \\
& +(1-\vartheta) \sum_{i=k+1}^{m} \int_{0}^{T}\left\|u_{m}\right\|_{\mathfrak{A}^{*}}^{2} d t, \quad \vartheta \in(0,1),
\end{aligned}
$$

and $z^{d}=\left(z_{1}^{d}, z_{2}^{d}, \ldots, z_{m}^{d}\right)$ is the required state of the system. Functional (13) is a compromise functional and allows to bring the desired solution $x$ closer to the required state $z^{d}$ at the lowest control costs. Problem (1) - (3), (7), (12) describes the optimal control problem of the propagation of a nerve impulse in the system of nerves and allows to achieve the desired system response for the least external impact.

Definition 2. Refer to a pair $(\tilde{x}, \tilde{u}) \in \mathfrak{X} \times \mathfrak{U}_{a d}$ as a solution to optimal control problem $(1)-(3),(7),(12)$ if

$$
J(\tilde{x}, \tilde{u})=\inf _{(x, u)} J(x, u),
$$

where the pairs $(x, u) \in \mathfrak{X} \times \mathfrak{U}_{a d}$ satisfy (1) - (3), (7) in the sense of Definition 1; the vector function $\tilde{u}$ is called the optimal control.

Theorem 2. [16] Suppose that $\alpha_{i} \in \mathbb{R}_{+}$for $i=\overline{1, m}$ and conditions (8) are satisfied. Then there exists a solution $(\tilde{x}, \tilde{u}) \in \mathfrak{X} \times \mathfrak{U}_{a d}$ to problem $(1)-(3)$, (7), (12) for every $x_{0} \in \mathfrak{A}$.

\section{Numerical Algorithm}

Based on the theoretical results obtained in the article [16], we develop an algorithm for the approximate solution of optimal control problem (1) - (3), (7), (12) based on the modified decomposition, Galerkin and Ritz methods. Applying the decomposition method described in [12], we linearize system (1) by introducing the function $y_{i}, i=\overline{1, k}$, and obtain equivalent Showalter - Sidorov problem (7) for the system of equations

$$
\left\{\begin{array}{c}
\left\langle v_{1 t}^{n}-\alpha_{1} v_{1 s s}^{n}+\beta_{11} v_{1}^{n}+\beta_{12} v_{2}^{n}+\cdots+\beta_{1 m} v_{m}^{n}+\left(y_{1}^{n}\right)^{3}, \varphi_{l}\right\rangle=\left\langle u_{1}, \varphi_{l}\right\rangle, \\
\left\langle v_{2 t}^{n}-\alpha_{2} v_{2 s s}^{n}+\beta_{21} v_{1}^{n}+\beta_{22} v_{2}^{n}+\cdots+\beta_{2 m} v_{m}^{n}+\left(y_{2}^{n}\right)^{3}, \varphi_{l}\right\rangle=\left\langle u_{2}, \varphi_{l}\right\rangle, \\
\cdots \\
\left\langle v_{k t}^{n}-\alpha_{k} v_{k s s}^{n}+\beta_{k 1} v_{1}^{n}+\beta_{k 2} v_{2}^{n}+\cdots+\beta_{k m} v_{m}^{n}+\left(y_{k}^{n}\right)^{3}, \varphi_{l}\right\rangle=\left\langle u_{k}, \varphi_{l}\right\rangle, \\
\left\langle-\alpha_{k+1} v_{(k+1) s s}^{n}+\beta_{(k+1) 1} v_{1}^{n}+\beta_{(k+1) 2} v_{2}^{n}+\cdots+\beta_{(k+1) m} v_{m}^{n}, \varphi_{l}\right\rangle=\left\langle u_{(k+1)}, \varphi_{l}\right\rangle, \\
\cdots \\
\left\langle-\alpha_{m} v_{m s s}^{n}+\beta_{m 1} v_{1}^{n}+\beta_{m 2} v_{2}^{n}+\cdots+\beta_{m m} v_{m}^{n}, \varphi_{l}\right\rangle=\left\langle u_{m}, \varphi_{l}\right\rangle, \\
v_{i j}^{n}=y_{i j}^{n}, i=\overline{1, k}, j=\overline{1, K}, l=\overline{1, n}, \text { for all } s \in\left(0, l_{j}\right) .
\end{array}\right.
$$


Then the solution $(x, u)$ to problem $(1)-(3),(7),(12)$ is reduced to the unknown triple $(x, y, u)$, where $x=\left(v_{1}, v_{2}, \ldots, v_{m}\right), y=\left(y_{1}, y_{2}, \ldots, y_{k}\right), u=\left(u_{1}, u_{2}, \ldots, u_{m}\right)$. Following the Galerkin method, we construct an approximate solution $\tilde{x}=\left(\tilde{v}_{1}, \tilde{v}_{2}, \ldots, \tilde{v}_{m}\right)$, $\tilde{y}=\left(\tilde{y}_{1}, \tilde{y}_{2}, \ldots, \tilde{y}_{k}\right)$ to problem $(2),(3),(7),(12),(14)$ in the form of the sums

$$
\tilde{v}_{i}(s, t)=v_{i}^{n}(s, t)=\sum_{l=1}^{n} v_{i}^{l}(t) \varphi_{l}(s), i=\overline{1, m}, \quad \tilde{y}_{i}(s, t)=y_{i}^{n}(s, t)=\sum_{l=1}^{n} y_{i}^{l}(t) \varphi_{l}(s), i=\overline{1, k} .
$$

Represent the right-hand side of the equation in the form

$$
\tilde{u}_{i}(s, t)=\sum_{l=1}^{n} u_{i}^{l}(t) \varphi_{l}(s), i=\overline{1, m}
$$

Using the Ritz method, we represent the unknown functions $y_{i}^{l}(t), i=\overline{1, k}, u_{i}^{l}(t), i=$ $\overline{1, m}, l=\overline{1, n}$, by the expansion $y_{i}^{l}(t, H)=\sum_{h=0}^{H} b_{h, i}^{l} t^{h}, u_{i}^{l}(t, H)=\sum_{h=0}^{H} c_{h, i}^{l} t^{h}$. In order to find the unknowns $v_{i}^{n}(t)$, use the Galerkin sums, and then multiply the resulting equation scalarly in $\mathcal{H}$ by the eigenfunctions $\varphi_{l}(s)$. As a result, we obtain the algebra-differential equations

$$
\left\langle L \tilde{x}_{t}, \varphi_{l}(s)\right\rangle+\left\langle M_{1} \tilde{x}, \varphi_{l}(s)\right\rangle+\left\langle M_{2}(\tilde{y}), \varphi_{l}(s)\right\rangle=\left\langle\tilde{u}, \varphi_{l}(s)\right\rangle, l=\overline{1, n},
$$

with Showalter-Sidorov conditions (10). Next, we construct an approximate solution to optimal control problem (2), (3), (7), (12), (14) by the penalty method described in [12]. Consider an equivalent optimal control problem, where the relation $v_{i}^{n} \rightarrow y_{i}^{n}, i=\overline{1, k}$ for $n \rightarrow \infty$ is obtained for an approximate solution by introducing a new functional of the form

$$
\begin{aligned}
& J(x, u)=\vartheta \theta \sum_{i=1}^{k} \int_{0}^{T}\left\|\tilde{v}_{i}-z_{i}^{d}\right\|_{L_{4}(\mathbf{G})}^{4} d t+\vartheta(1-\theta) \sum_{i=1}^{k} \int_{0}^{T}\left\|\tilde{y}_{i}-z_{i}^{d}\right\|_{L_{4}(\mathbf{G})}^{4} d t+ \\
& +\vartheta \sum_{i=k+1}^{m} \int_{0}^{T}\left\|\tilde{v}_{i}-z_{i}^{d}\right\|_{\mathfrak{A}}^{2} d t+(1-\vartheta) \sum_{i=1}^{k} \int_{0}^{T}\left\|\tilde{u}_{m}\right\|_{L_{\frac{4}{3}}(\mathbf{G})}^{\frac{4}{3}} d t+(1-\vartheta) \sum_{i=k+1}^{m} \int_{0}^{T}\left\|\tilde{u}_{i}\right\|_{\mathfrak{A}^{*}}^{2} d t+ \\
& +r_{\varepsilon} \sum_{i=1}^{k} \int_{0}^{T}\left\|\tilde{v}_{i}-\tilde{y}_{i}\right\|_{L_{4}(\mathbf{G})}^{4} d t, \theta \in(0,1), \quad \vartheta \in(0,1), \quad(16)
\end{aligned}
$$

where the penalty parameter $r_{\varepsilon}=\frac{1}{\varepsilon} \rightarrow+\infty$ for $\varepsilon \rightarrow+0$.

Based on the theoretical results obtained, a program for numerical solution of problem (1) - (3), (7), (12) was developed and implemented in the Maple 17.0 for Windows environment in Maple programming language. The result of the program is shown in Example.

Example. Consider problem (1) - (3), (7), (12) on the graph G (see Fig. 1) consisting of three consecutively joined edges and four vertices, $d_{1}=d_{2}=1, l_{1}=l_{2}=\pi$ in the case 


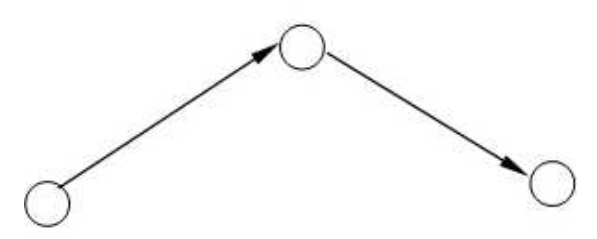

Fig. 1. The graph $G$

of $k=1, m=2, K=2, n=2, \alpha_{1}=2, \alpha_{2}=1, \beta_{11}=1, \beta_{12}=1, \kappa=1, \beta_{21}=1$, $\beta_{22}=1, T=1, \theta=\frac{1}{2}, \vartheta=\frac{99}{100}, \varepsilon=\frac{1}{100}, z_{11}^{d}(s, t)=\sqrt{\frac{1}{\pi}} t \cos \frac{s}{2}, z_{12}^{d}(s, t)=\sqrt{\frac{1}{\pi}} \frac{1}{2} t \cos \frac{s}{2}$, $z_{21}^{d}=-\sqrt{\frac{1}{\pi}} t \sin \frac{s}{2}, z_{22}^{d}=-\sqrt{\frac{1}{\pi}} t \sin \frac{s}{2}$, and the initial functions

$$
v_{011}(s)=\sqrt{\frac{1}{\pi}} \cos \frac{s}{2}, v_{012}(s)=-\sqrt{\frac{1}{\pi}} t \sin \frac{s}{2} .
$$

We apply the decomposition method and proceed to the problem

$$
\left\{\begin{array}{l}
v_{1 j t}-2 v_{1 j s s}+v_{1 j}+v_{2 j}+y_{j}^{3}=u_{1 j}, \\
-v_{2 j s s}-v_{1 j}+v_{2 j}=u_{2 j}, j=1,2,
\end{array}\right.
$$

with the continuity condition and the flow balance condition

$$
\begin{aligned}
& v_{1 s}(\pi, t)=v_{2 s}(0, t), v_{1 s}(0, t)=0 \\
& v_{2 s}(\pi, t)=0, v_{1}(\pi, t)=v_{2}(0, t)
\end{aligned}
$$

On the graph $\mathbf{G}$, the eigenfunctions of the Sturm-Liouville problem

$$
\begin{gathered}
X_{1}^{\prime \prime}=\lambda X_{1}, X_{2}^{\prime \prime}=\lambda X_{2}, \\
X_{1}^{\prime}(\pi)=X_{2}^{\prime}(0)=0, X_{1}(\pi)=X_{2}(0), \\
X_{1}^{\prime}(0)=0, X_{2}^{\prime}(\pi)=0
\end{gathered}
$$

have the form [18]

$$
\begin{gathered}
\varphi_{1}=\left(\varphi_{1}^{1}, \varphi_{1}^{2}\right)=\left(\sqrt{\frac{1}{\pi}}, \sqrt{\frac{1}{\pi}}\right), \\
\varphi_{2}=\left(\varphi_{2}^{1}, \varphi_{2}^{2}\right)=\left(\frac{1}{\pi} \cos \frac{s}{2}, \frac{1}{\pi} \cos \frac{s}{2}\right) .
\end{gathered}
$$

As a result of calculations, we find the control coefficients shown in Table, for which the value of the functional is $J=0,18162266$ (accurate to $10^{-8}$ ), and the solution to the problem is shown in Figs. $2-6$. We show changes in concentrations in the first link of the tubular reactor in $(0, \pi)$, in the second link of the system of nerves in $(\pi, 2 \pi)$, and in the third link of the system of nerves in $(2 \pi, 3 \pi)$. 
Table

The control coefficients (accurate to $10^{-8}$ )

\begin{tabular}{|c|c|c|c|}
\hline$b_{1,1}=0,00390231$ & $b_{1,2}=-0,00471158$ & $b_{2,1}=-0,00678036$ & $b_{2,2}=-0,00891264$ \\
\hline$c_{1,0}^{1}=0,00000514$ & $c_{1,1}^{1}=-0,00370847$ & $c_{1,2}^{1}=0,00460690$ & $c_{2,0}^{1}=0,00004497$ \\
\hline$c_{2,1}^{1}=0,00671085$ & $c_{2,2}^{1}=-0,00900376$ & $c_{1,0}^{2}=0,00398598$ & $c_{1,1}^{2}=-0,00587379$ \\
\hline$c_{1,2}^{2}=-0,00425381$ & $c_{2,0}^{2}=-0,00689106$ & $c_{2,1}^{2}=0,00992757$ & $c_{2,2}^{2}=0,01041112$ \\
\hline
\end{tabular}

The difference between the obtained solution $x=\left(v_{1}, v_{2}\right)$ and the required solution $y$ is as follows:

$$
\begin{aligned}
& \Delta_{1}=\left(\int_{0}^{T}\left\|v_{1}(s, t)-y(s, t)\right\|_{L_{4}(\mathbf{G})}^{4} d t\right)^{\frac{1}{4}}=2,2986301 \cdot 10^{-8} \\
& \Delta_{2}=\left(\int_{0}^{T}\left\|v_{1}(s, t)-z_{1}^{d}(s, t)\right\|_{L_{4}(\mathbf{G})}^{4} d t\right)^{\frac{1}{4}}=0,019204719 \\
& \Delta_{3}=\left(\int_{0}^{T}\left\|y(s, t)-z_{d}^{1}(s, t)\right\|_{L_{4}(\mathbf{G})}^{4} d t\right)^{\frac{1}{4}}=0,019194064 \\
& \Delta_{4}=\left(\int_{0}^{T}\left\|v_{2}(s, t)-z_{2}^{d}(s, t)\right\|_{L_{4}(\mathbf{G})}^{4} d t\right)^{\frac{1}{4}}=0,0011103445
\end{aligned}
$$

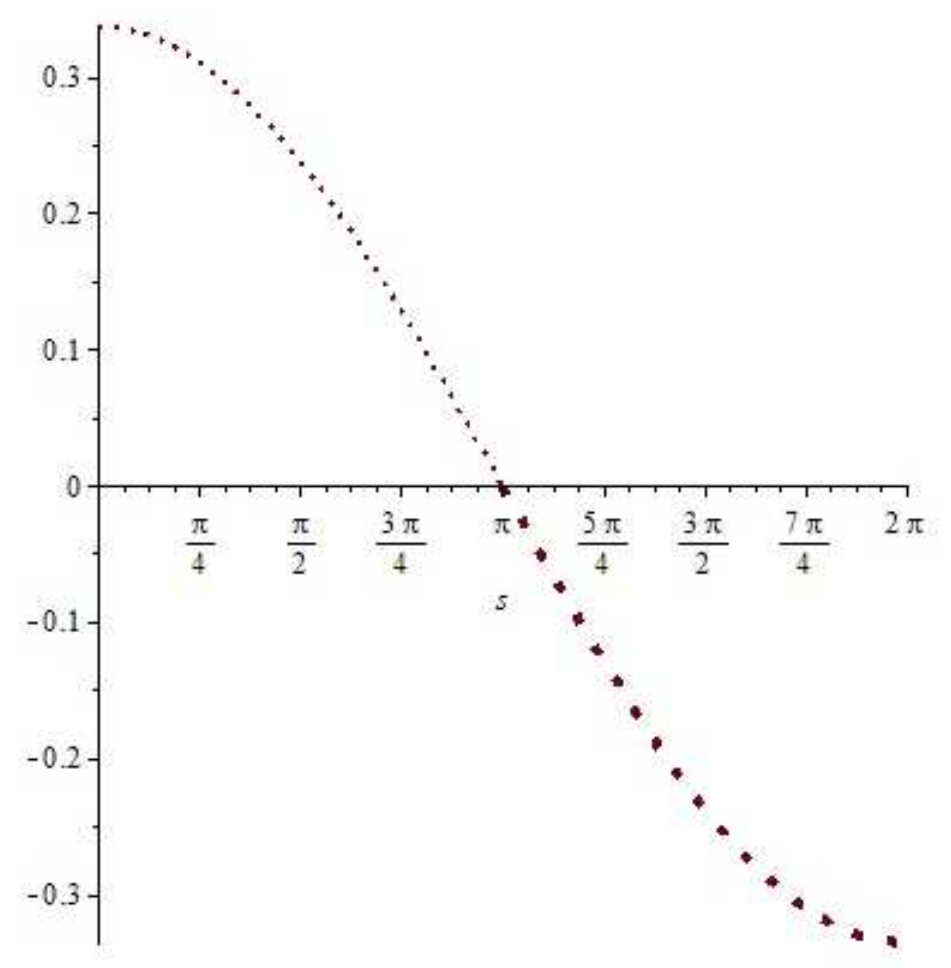

Fig. 2. Graphs of the optimal control $u_{1 j}(s, 1)$ for $t=1, j=1,2$ 


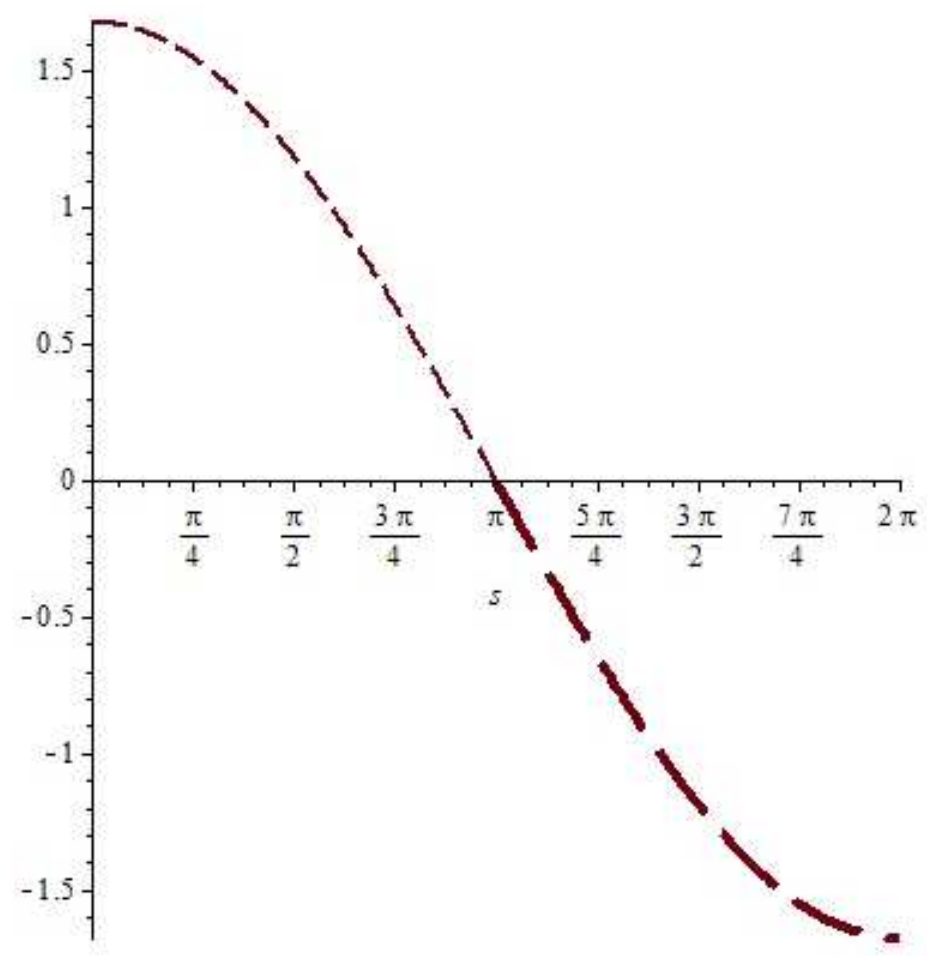

Fig. 3. Graphs of the optimal control $u_{2 j}(s, 1)$ for $t=1, j=1,2$

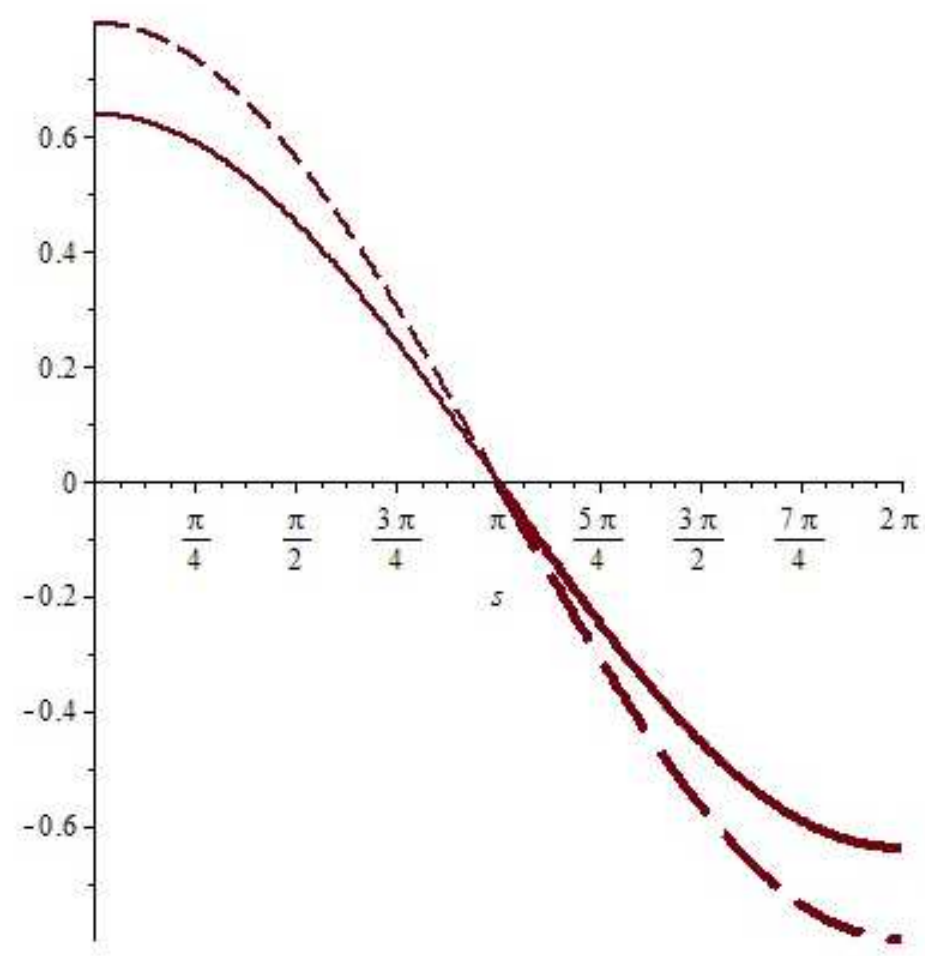

Fig. 4. Graphs of $v_{1 j}(s, 1), z_{1 j}^{d}(s, 1)$ for $t=1, j=1,2$ 


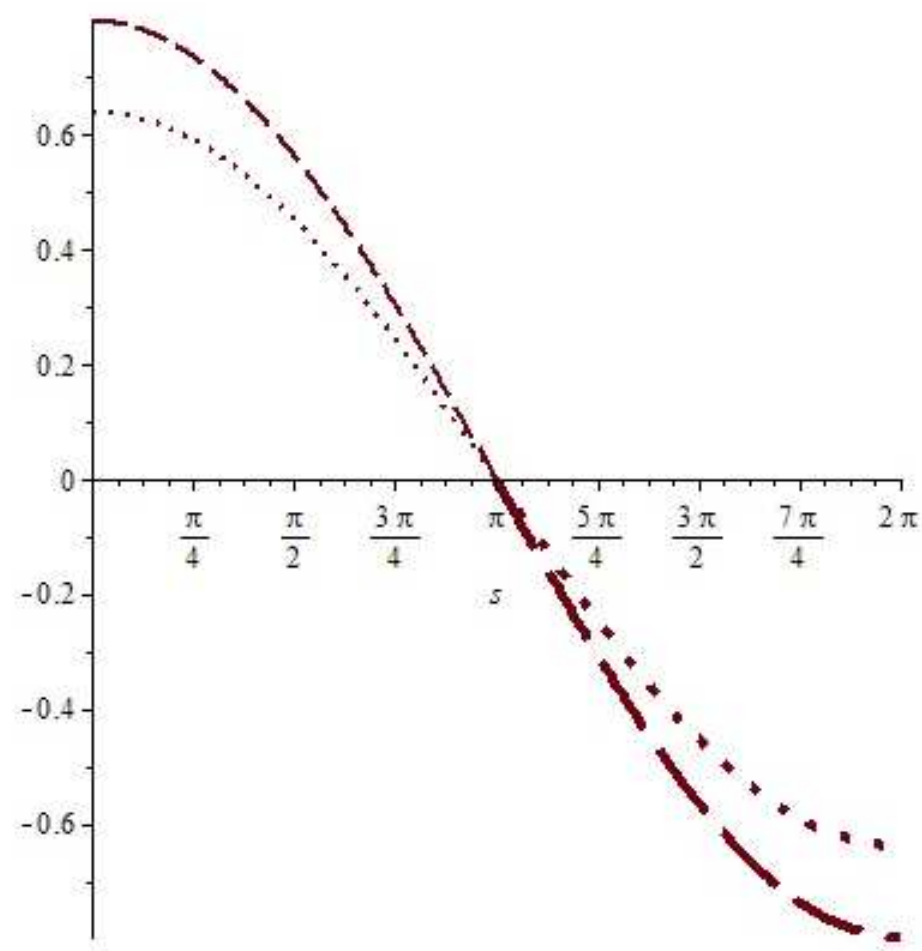

Fig. 5. Graphs of $y_{j}(s, 1), z_{1 j}^{d}(s, 1)$ for $t=1, j=1,2$

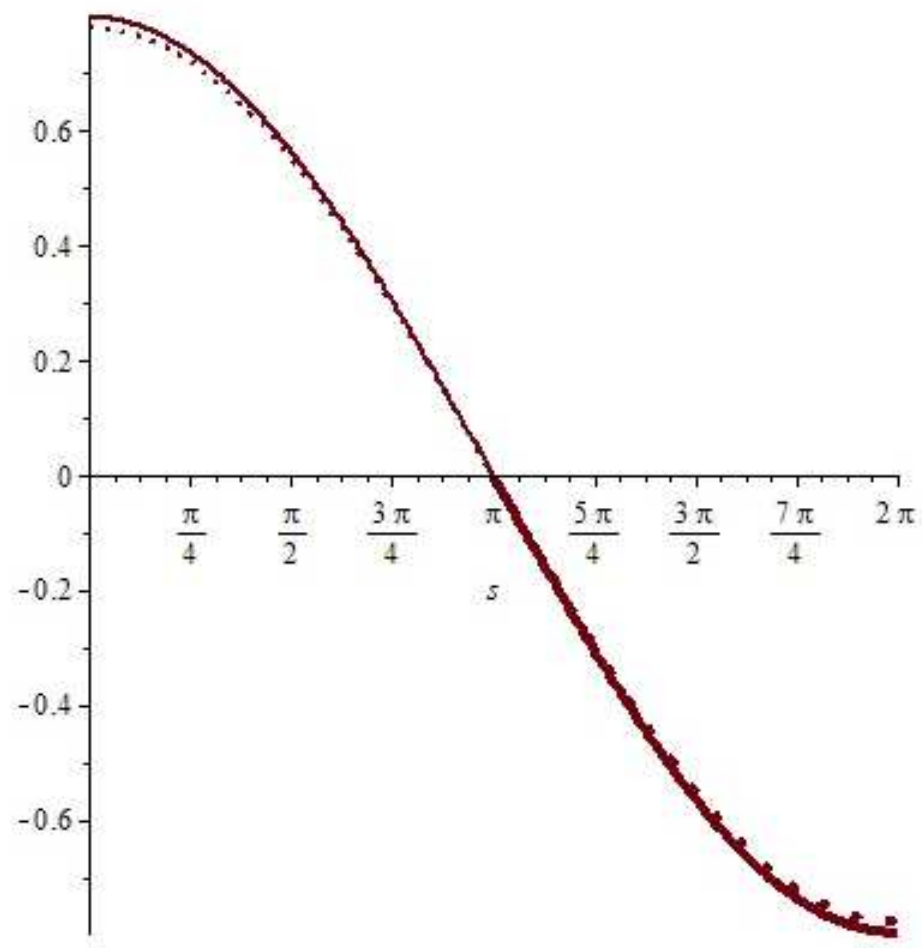

Fig. 6. Graphs of $v_{2 j}(s, 1), z_{2 j}^{d}(s, 1)$ for $t=1, j=1,2$ 


\section{References}

1. Hodgkin A. L., Huxley A. F. A Quantitative Description of Membrane Current and Its Application to Conduction and Excitation in Nerve. The Journal of Physiology, 1952, no. 117 (4), pp. 500-544.

2. Lefever R., Prigogin I. Symmetry-Breaking Instabilities in Dissipative System. II. American Journal of Physics, 1968, vol. 48, pp. 1695-1700.

3. Fitz Hugh R. Mathematical Models of Threshold Phenomena in the Nerve Membrane. Bulletin of Mathematical Biology, 1955, vol. 17, no. 4, pp. 257-278.

4. Nagumo J., Arimoto S., Yoshizawa S. An Active Pulse Transmission Line Simulating Nerve Axon. Proceedings of the IRE, 1962, vol. 50, no. 10, pp. 2061-2070.

5. Gubernov V. V., Kolobov A. V., Polezhaev A. A. et. al. Stabilization of Combustion Wave Through the Competitive Endothermic Reaction. Proceeding of the Royal Society A, 2015, vol. 471, no. 2180, article ID: 20150293.

6. Weber S., Porto M. Multicomponent Reaction-Diffusion Processes on Complex Networks. Physical Review E, 2006, vol. 74, no. 4, pp. 046108.

7. Bokareva T. A., Sviridyuk G. A. Whitney Folds of the Phase Spaces of Some Semilinear Equations of Sobolev Type. Mathematical Notes, 1994, vol. 55, no. 3-4, pp. 237-242.

8. Sviridyuk G. A., Shemetova V. V. Hoff Equations on Graphs. Differential Equations, 2006, vol. 42, no. 1, pp. 139-145. DOI: 10.1134/S0012266106010125

9. Sviridyuk G. A., Efremov A. A. Optimal Control of Sobolev Type Linear Equations with Relativity p-Sectorial Operators. Differential Equations, 1995, vol. 31, no. 11, pp. 1882-1890.

10. Sagadeeva M. A., Zagrebina S. A., Manakova N. A. Optimal Control of Solutions of a Multipoint Initial-Final Problem for Non-Autonomous Evolutionary Sobolev Type Equation. Evolution Equations and Control Theory, 2019, vol. 8, no. 3, pp. 473-488. DOI: 10.3934 /eect.2019023

11. Zamyshlyaeva A. A., Tsyplenkova O. N. Optimal Control of Solutions of the ShowalterSidorov-Dirichlet Problem for the Boussinesq-Love Equation. Differential Equations, 2013, vol. 49, no. 11, pp. 1356-1365. DOI: 10.1134/S0012266113110049

12. Manakova N. A. Method of Decomposition in the Optimal Control Problem for Semilinear Sobolev Type Models. Bulletin of the South Ural State University. Series: Mathematical Modelling, Programming and Computer Software, 2015, vol. 8, no. 2, pp. 133-137. DOI: 10.14529/mmp150212 (in Russian)

13. Sviridyuk G. A., Manakova N. A. An Optimal Control Problem for the Hoff Equation. Journal of Applied and Industrial Mathematics, 2007, vol. 1, no. 2, pp. 247-253.

14. Ryll Ch., Lober J., Martens St. et. al. Analytical, Optimal, and Sparse Optimal Control of Traveling Wave Solutions to Reaction-Diffusion Systems. Control of Self-Organizing Nonlinear Systems, 2016, pp. 189-210.

15. Manakova N. A., Gavrilova O. V. Optimal Control for a Mathematical Model of Nerve Impulse Spreading. Bulletin of the South Ural State University. Series: Mathematical Modelling, Programming and Computer Software, 2015, vol. 8, no. 4, pp. 120-126. DOI: $10.14529 / \mathrm{mmp} 150411$ 
16. Gavrilova O. V. Optimal Control by Solutions of a Multicomponent Model of ReactionDiffusion in a Tubular Reactor. Bulletin of the South Ural State University. Series: Mathematics. Mechanics. Physics, 2020, vol. 12, no. 1, pp. 14-23. DOI: $10.14529 / \mathrm{mmph} 200102$

17. Sviridyuk G. A. [On the Solvability of Singular Systems of Ordinary Differential Equations]. Differentsial'nye Uravneniya [Differential Equations], 1987, vol. 23, no. 9, pp. 1637-1639. (in Russian)

18. Zamyshlyaeva A. A., Lut A. V. Numerical Investigation of the Boussinesq-Love Mathematical Models on Geometrical Graphs, Bulletin of the South Ural State University. Series: Mathematical Modelling, Programming and Computer Software, 2017, vol. 10, no. 2, pp. 137-143. DOI: 10.14529/mmp170211

19. Bayazitova A. A. The Sturm-Liouville Problem on Geometric Graph. Bulletin of the South Ural State University. Series: Mathematical Modelling, Programming and Computer Software, 2010, no. 16 (192), issue 5, pp. 4-10. (in Russian)

Olga V. Gavrilova, PhD Student, Department of Mathematical Physics Equations, South Ural State University, Chelyabinsk, Russian Federation, gavrilovaov@susu.ru.

\title{
ЧИСЛЕННОЕ ИССЛЕДОВАНИЕ ЗАДАЧИ
}

ОПТИМАЛЬНОГО УПРАВЛЕНИЯ ДЛЯ ВЫРОЖДЕННОЙ МНОГОКОМПОНЕНТНОЙ МАТЕМАТИЧЕСКОЙ МОДЕЛИ РАСПРОСТРАНЕНИЯ НЕРВНОГО ИМПУЛЬСА В СИСТЕМЕ НЕРВОВ

\section{О. В. Гаврилова}

\begin{abstract}
Статья посвящена численному исследованию многокомпонентной модели задачи оптимального регулирования распространением нервного импульса в системе нервов на основе системы уравнений Фитцхью - Нагумо. Эта модель относится к классу моделей реакции-диффузии, которые моделируют широкий класс процессов, таких как химические реакции с диффузией и распространение нервного импульса. В случае асимптотической устойчивости исследуемой модели и при условии, что скорость изменения одних компонент значительно превышает скорость других, исследуемая задача может быть сведена к задаче оптимального управления для полулинейного уравнения соболевского типа с начальным условием Шоуолтера - Сидорова. В статье разработан алгоритм численного исследования задачи в среде Maple. Алгоритм основан на методе Галеркина и методе декомпозиции, что позволяет учесть вырожденность уравнения. В статье приведен пример, иллюстрирующих результаты вычислительного эксперимента, полученные для двухкомпонентной модели на двухреберном графе.

Ключевые слова: уравнения соболевского типа; задача Шоуолтера - Сидорова; задача оптимального управления.
\end{abstract}




\section{Литература}

1. Hodgkin, A.L. A Quantitative Description of Membrane Current and Its Application to Conduction and Excitation in'Nerve / A. L. Hodgkin, A. F. Huxley // The Journal of Physiology. - 1952. - № 117 (4). - P. 500-544.

2. Lefever, R. Symmetry-Breaking Instabilities in Dissipative System. II / R. Lefever, I. Prigogin // The Journal of Chemical Physics. - 1968. - V. 48, № 4. - P. 1695-1700.

3. Fitz Hugh, R. Mathematical Models of Threshold Phenomena in the Nerve Membrane / R. Fitz Hugh // Bulletin of Mathematical Biology. - 1955. - V. 17, № 4. - P. 257-278.

4. Nagumo, J. An Active Pulse Transmission Line Simulating Nerve Axon / J. Nagumo, S. Arimoto, S. Yoshizawa // Proceedings of the IRE. - 1962. - V. 50, № 10. P. 2061-2070.

5. Gubernov, V. V. Stabilization of Combustion Wave Through the Competitive Endothermic Reaction / V. V. Gubernov, A. V. Kolobov, A. A. Polezhaev et. al. // Proceeding of the Royal Society A. - 2015. - V. 471, № 2180. - P. 20150293.

6. Weber, S. Multicomponent Reaction-Diffusion Processes on Complex Networks S. Weber, M. Porto // Physical Review E. - 2006. - V. 74, № 4. - P. 046108.

7. Bokareva, T. A. Whitney Folds of the Phase Spaces of Some Semilinear Equations of Sobolev Type / T. A. Bokareva, G. A. Sviridyuk // Mathematical Notes. - 1994. V. 55, № 3-4. - P. 237-242.

8. Sviridyuk, G. A. Hoff Equations on Graphs / G. A. Sviridyuk, V. V. Shemetova // Differential Equations. - 2006. - V. 42, № 1. - P. 139-145.

9. Sviridyuk, G. A. Optimal Control of Sobolev Type Linear Equations with Relativity p-Sectorial Operators / G. A. Sviridyuk, A. A. Efremov // Differential Equations. 1995. - V. 31, № 11. - P. 1882-1890.

10. Sagadeeva, M. A. Optimal Control of Solutions of a Multipoint Initial-Final Problem for Non-Autonomous Evolutionary Sobolev Type Equation / M. A. Sagadeeva, S. A. Zagrebina, N. A. Manakova // Evolution Equations and Control Theory. 2019. - V. 8, № 3. - P. 473-488.

11. Zamyshlyaeva, A. A. Optimal Control of Solutions of the Showalter-SidorovDirichlet Problem for the Boussinesq-Love Equation / A. A. Zamyshlyaeva, O. N. Tsyplenkova // Differential Equations. - 2013. - V. 49, № 11. - P. 1356-1365.

12. Манакова, Н. А. Метод декомпозиции в задаче оптимального управления для полулинейных моделей соболевского типа / Н. А. Манакова // Вестник ЮУрГУ. Серия: Математическое моделирование и программирование. - 2015. - Т. 8, № 2. C. $133-137$.

13. Sviridyuk, G. A. An Optimal Control Problem for the Hoff Equation / G. A. Sviridyuk, N. A. Manakova // Journal of Applied and Industrial Mathematics. - 2007. - V. 1, № 2. - P. 247-253. 
14. Ryll, Ch. Analytical, Optimal, and Sparse Optimal Control of Traveling Wave Solutions to Reaction-Diffusion Systems / Ch. Ryll, J. Lober, St. Martens et. al. // Control of Self-Organizing Nonlinear Systems. - 2016. - P. 189-210.

15. Manakova, N. A. Optimal Control for a Mathematical Model of Nerve Impulse Spreading / N. A. Manakova, O. V. Gavrilova // Вестник ЮУрГУ. Серия: Математическое моделирование и программирование. - 2015. - Т. 8, № 4. - С. 120-126.

16. Gavrilova, O. V. Optimal Control By Solutions Of A Multicomponent Model Of Reaction-Diffusion In A Tubular Reactor / О. V. Gavrilova // Вестник ЮУрГУ. Серия: Математика. Механика. Физика. - 2020. - V. 12, № 1. - Р. 14-23.

17. Свиридюк, Г. А. Об одной сингулярной системе обыкновенных дифференциальных уравнений / Г. А. Свиридюк // Дифференциальные уравнения. - 1987. T. 23, № 9. - C. 1637-1639.

18. Zamyshlyaeva, A. A. Numerical Investigation of the Boussinesq-Love Mathematical Models on Geometrical Graphs / A. A. Zamyshlyaeva, A. V. Lut // Вестник ЮУрГУ. Серия: Математическое моделирование и программирование. - 2017. - Т. 10, № 2.C. $137-143$.

19. Баязитова, А. А. Задача Штурма-Лиувилля на геометрическом графе / А. А. Баязитова // Вестник ЮУрГУ. Серия: Математическое моделирование и программирование. - 2010. - № 16 (192). - С. 4-10.

Гаврилова Ольга Витальевна, аспирант, кафедра уравнений математической физики, Южно-Уральский государственный университет (г. Челябинск, Российская Федераuия),gavrilovaov@susu.ru.

Поступила в редакцию 30 января 2020 г. 